\title{
Fever Management in Sickle Cell Disease in Low- and Middle-Income Countries: A Survey of SCD Management Programs
}

\author{
Alexandra L. Coria, ${ }^{1}$ Catherine M. Taylor, ${ }^{2}$ and Venée N. Tubman ${ }^{3,4 *}$ \\ ${ }^{1}$ Maimonides Children's Hospital, Brooklyn, New York; ${ }^{2}$ Lewisham and Greenwich NHS Trust, London, United Kingdom; ${ }^{3}$ Department of \\ Pediatrics, Baylor College of Medicine, Houston, Texas; ${ }^{4}$ Texas Children's Cancer and Hematology Centers, Houston, Texas
}

\begin{abstract}
Management of fever is a key element of care for children with sickle cell disease (SCD). There exist few studies of current practices in managing fevers in SCD in low- and middle-income countries (LMICs) and malaria-endemic regions where SCD is prevalent. We surveyed medical providers in these settings to characterize current practices in infection prevention and fever management for children with SCD. We found wide variation in use of newborn screening for early diagnosis and infection prevention, pneumococcal vaccination, use of antibiotics and antimalarials, and route of antibiotic administration. Counter to established guidelines, $78 \%$ (95\% Cl: $59-100 \%)$ of respondents would consider using oral antibiotics for a febrile child with SCD. Only $17 \%(95 \% \mathrm{Cl}$ : $0-37 \%)$ would administer antibiotics to a wellappearing child with a positive malaria test. Availability of blood cultures did not affect duration of antibiotic course. Further study and standardization of fever management in SCD in LMICs are urgently needed.
\end{abstract}

More than 300,000 infants are born with sickle cell disease (SCD) each year, making it the most prevalent monogenetic disease in the world. ${ }^{1}$ Guidelines for the management of fever in SCD have been published for both high-income countries $(\mathrm{HICs})^{2}$ and low- and middle-income countries (LMICs). ${ }^{3,4}$ Infection by encapsulated organisms is a known cause of mortality in the United States ${ }^{5,6}$ and is equally important in sub-Saharan Africa., ${ }^{3,7}$ Prevention of infection-related mortality in SCD requires preventive management (e.g. immunization and antimicrobial prophylaxis) and acute management of febrile illness. Acute management should include urgent administration of antibiotics targeting encapsulated bacteria and guiding treatment with blood cultures if available. In the WHO's African region, where $77 \%$ of SCD births occur, ${ }^{1}$ malaria threatens childhood survival and poses a specific threat to children with SCD.

In many LMICs, tools for preventive management and acute management may be limited because of resource constraints. Guidelines must be tailored locally to account for these constraints. To refine existing recommendations and to design rational, evidence-based fever management guidelines for patients with SCD in LMICs, it is important to understand how challenges specific to LMICs are currently addressed by SCD programs.

The objective of this study was to describe the management of febrile illness in children with SCD in LMICs. We designed a 40-question survey using an iterative methodology, expanding upon standard practices established in the United States ${ }^{2}$ with additional consideration of issues specific to malariaendemic regions. The survey was distributed OctoberDecember 2015. It was published in REDCap and distributed to health-care providers at international conferences and via email, in French and English. Data were tabulated and analyzed using Microsoft Excel 2008 (Microsoft Corp., Redmond, WA). $P$-values were generated using one-tailed Fisher's exact test. The surveys did not require answers to all questions; therefore, we report the number of respondents (" $n$ ") for each result reported in the following paragraphs. Cls were calculated using the Clopper-Pearson exact method. This survey

*Address correspondence to Venée N. Tubman, Texas Children's Cancer and Hematology Centers, Feigin Center, Ste. 1025, 1102 Bates St., Houston, TX 77030. E-mail: vntubman@txch.org was exempted from ethical review by the Boston Children's Hospital Institutional Review Board.

Twenty-eight surveys that returned from unique programs in 13 LMICs, including nine malaria-endemic countries, were included in the analysis. Ninety-three percent of individual respondents were physicians, all of whom practice in the country represented. Thirteen programs (46\%) served fewer than 250 patients, and six (21\%) programs served more than 1,000 patients. Three programs that did not routinely treat patients younger than 17 years were excluded from analyses of pneumococcal conjugate vaccine (PCV) use and malaria prophylaxis practices.

Programs operationalized the management of fever using varied approaches. Fifty-four percent of respondents had written fever management protocols. Among 27 individual respondents, 33\% (95\% Cl: 17-54\%) defined a fever as $37.5^{\circ} \mathrm{C}$ or higher, $59 \%(95 \% \mathrm{Cl}: 39-78 \%)$ as $38.0^{\circ}$, and $19 \%$ (95\% Cl: 6-38\%) as a higher number. Ninety-three percent (95\% Cl: 77-98\%) of respondents "always" or "almost always" had thermometers available in clinic.

Antimicrobial prophylaxis practices also varied. Thirty-two percent (95\% Cl: 16-52\%) of 28 respondents had newborn screening available, facilitating early initiation of penicillin prophylaxis. Of 24 respondents, 67\% (95\% Cl: 45-84\%) administered penicillin prophylaxis to all children younger than 5 years. Twenty-nine percent (95\% Cl: 13-51\%) never administered penicillin prophylaxis in their SCD clinic. Of 21 respondents in malarial regions, $76 \%$ (95\% Cl: 53-92\%) administered pharmaceutical malarial prophylaxis.

Immunization practices varied according to availability of vaccines. Thirty-nine percent (95\% Cl: $20-61 \%)$ of pediatric programs $(n=23)$ did not vaccinate with a PCV because no form of the vaccine was available. PCV vaccination either through the clinic or the national immunization program was provided in $57 \%$ (95\% Cl: $34-77 \%)$ of respondents' programs. The remaining $4 \%(95 \% \mathrm{Cl}: 0-24 \%)$ of programs vaccinated with PCV only if parents could afford it.

Treatment decisions for acute management of febrile illness may be impacted by antimicrobial treatments given before hospital presentation. Therefore, we explored provider perceptions of prehospital medication and self-administration. Of 27 respondents, $48 \%$ (95\% Cl: 29-68\%) estimated that more 
than half of their patients would self-administer antibiotics before presenting to a medical facility with a fever. In malariaendemic areas $(n=24), 71 \%$ (95\% Cl: 49-87\%) of respondents estimated that more than half of their patients self-administer antimalarials before presenting to a medical facility. On presentation, $72 \%$ (95\% Cl: $51-88 \%)$ of respondents $(n=25)$ said that they would "always" or "almost always" administer antibiotics to a febrile patient with SCD.

The potential for malaria infection confounds the standard response to fever in SCD. We explored the impact of malaria test result on antibacterial prescribing practices. Of 24 respondents from malaria-endemic regions, $79 \%(95 \% \mathrm{Cl}$ : 58-93\%) would "always" or "almost always" administer empiric antimalarials to a febrile patient with SCD. Only $17 \%$ (95\% Cl: $5-37 \%, n=24$ ) would administer antibiotics with antimalarials to a well-appearing child with a positive malaria test.

Empiric antibiotic administration is critical to management of febrile illness. Because intravenous antibiotic administration requires significant resources, we investigated provider preferences regarding route of administration. Of 26 respondents who answered follow-up questions regarding antibiotic choice, $31 \%$ (95\% Cl: $14-52 \%)$ said that oral antibiotics would be their first choice in an otherwise well-appearing patient (Figure 1). The choice of antibiotic used varied widely (Figure 2). Of 27 respondents to a question specifically about administration of oral antibiotics, $78 \%$ (95\% Cl: 59-91\%) would start with oral antibiotics in some situations. A majority of those whose first choice is a parenteral antibiotic would switch to oral within 1-3 days. The total duration of antibiotic therapy, whether oral or IV plus oral, ranged from 2 to 14 days, with a median and a mean of 7 days. Overall, $52 \%(95 \% \mathrm{Cl}$ : $32-71 \%)$ of programs $(n=27)$ had blood cultures available "always" or "almost always" at their facility or in a community laboratory. Among respondents who gave fewer than 5 total days of antibiotics, $100 \%$ (95\% Cl: 48-100\%) had blood cultures available "always" or "almost always." Among respondents who would give 5 or more days of antibiotics, only $41 \%$ (95\% Cl: $21-64 \%)$ had reliable access to blood cultures. Shorter antibiotic course correlated significantly $(p=0.02)$ with reliable access to blood cultures.

In this report, we describe the scope of preventive and acute management of febrile illness for patients with SCD in LMICs.

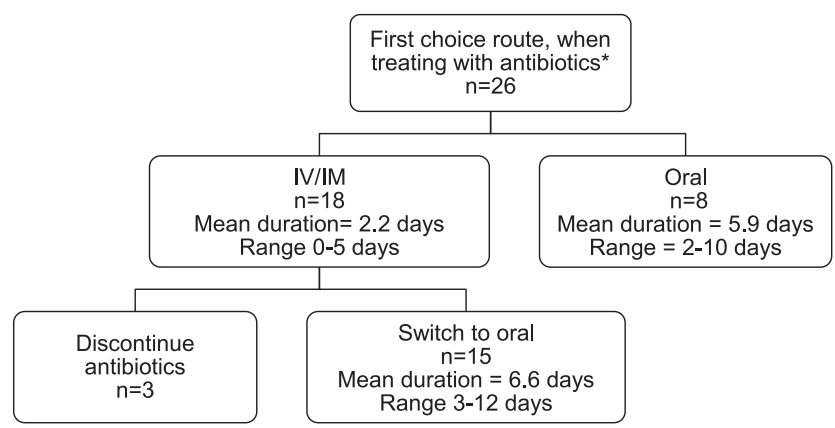

FIGURE 1. Preferred route and treatment course for first-line antibiotic regimen in patients with sickle cell disease (SCD) presenting with fever. Antibiotic regimen assumes patient is improving. *NB: $28 \%$ of respondents replied that they would treat febrile patients with SCD with antibiotics "almost never" or "half the time." These answers reflect what they would do if they DID opt for antibiotic administration.
Practice varied substantially across all areas surveyed. There was variation in how a fever was defined, possibly reflecting differences between published SCD literature and non-SCD pediatric literature., ${ }^{8,9}$ Written fever management protocols were used by just more than half of respondents' programs, signifying an opportunity to standardize management within programs.

Preventive measures begin to vary at birth. We affirmed the low prevalence of newborn screening across LMICs, although in several LMICs, newborn screening (NBS) has been widely credited with reducing mortality by facilitating early diagnosis and initiation of antibacterial prophylaxis. ${ }^{10-12}$ Availability of a PCV determined its use in our study. After diagnosing SCD, we observed variable use of penicillin prophylaxis. More respondents administered malarial chemoprophylaxis than bacterial prophylaxis despite clear guidelines for antibacterial prophylaxis. ${ }^{4}$ Although prophylaxis against malaria infection is recommended, there is ongoing debate regarding agent, schedule, and practicality of long-term chemoprophylaxis versus barrier prophylaxis., ${ }^{3,13}$ Future studies should investigate the outcomes of patients treated with antibacterial prophylaxis and various pharmaceutical or environmental forms of malarial prophylaxis and reasons for poor uptake of antibacterial prophylaxis.

Most respondents, when prescribing treatment antibiotics, do not conform to current guidelines, which recommend broad coverage targeting encapsulated organisms (Figure 2). ${ }^{2,4}$ Similar organisms (Streptococcus pneumoniae and gram-negative enteric organisms) are likely to cause infection in SCD in both HICs and LMICs. ${ }^{713-16}$ The variation in antibiotic choice may be due to antibiotic availability, perceptions of antibiotic pretreatment before presentation at a health facility, local culture data, or other factors which require further study. Although blood culture availability was associated with antibiotic duration, our sample size was too small to determine effect on antibiotic choice.

Most respondents would consider giving only oral antibiotics to a well-appearing febrile patient, although guidelines recommend parenteral therapy. In malarial regions, if a malaria test is positive, most respondents would consider not giving antibiotics, despite limited understanding of the influence of malaria on mortality in SCD ${ }^{17-19}$ and evidence that malaria and serious bacterial infections can be coincident. ${ }^{20}$ Updated guidelines should highlight the specific risk encapsulated organisms pose to children with SCD in settings with malaria to increase likelihood of antibiotics being given.

Although this study highlighted many areas for further study, we faced several limitations. Multivariate statistical analysis of the data was limited by a small sample size and variable completeness of the surveys. Although we presumed that resource limitations would drive deviation from published guidelines, the study questions were not designed to capture the influence of specific resource limitations on decisionmaking, except for blood cultures. For instance, antibiotic choice is likely influenced by local availability and cost. Finally, respondents were categorized as working in countries with or without malaria without accounting for within-country epidemiologic variation.

Our data support an urgent need to deepen the evidence base and training around practices for fever management in SCD in malaria-prone regions and in LMICs. Patient outcomes under current practices should be studied, to inform the development of a common set of principles of fever management 


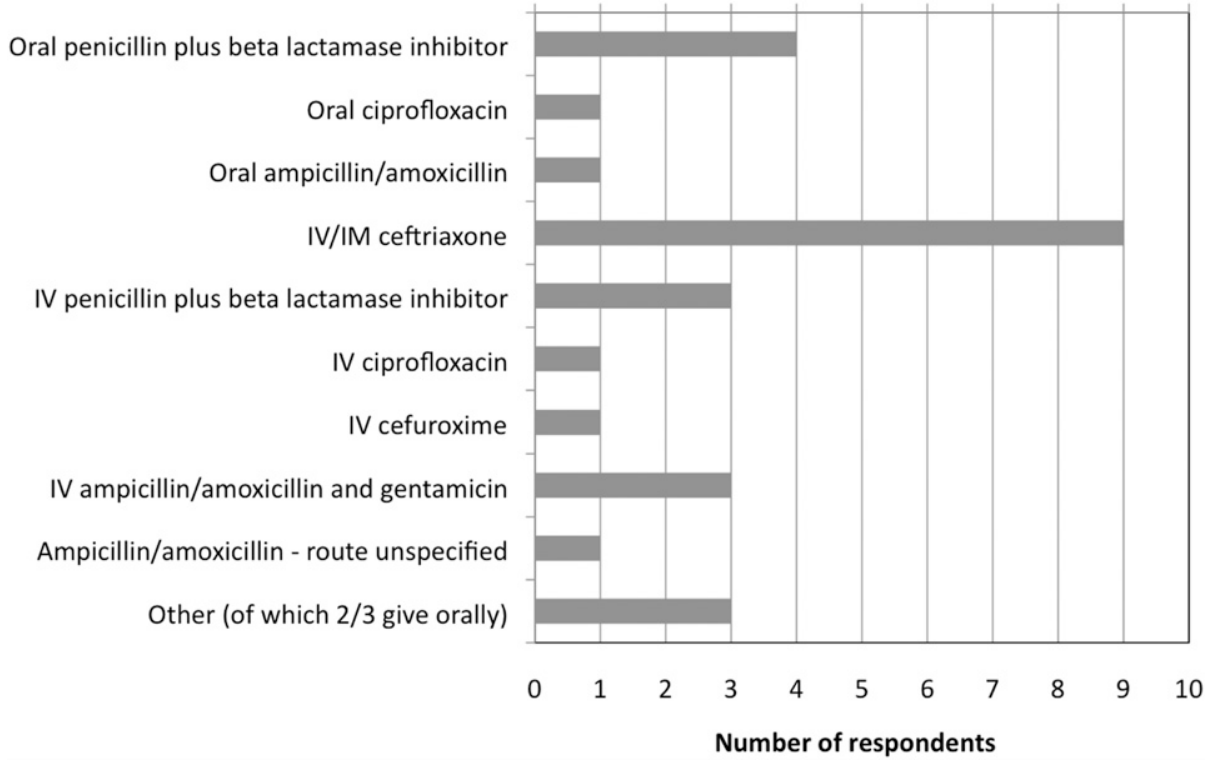

FIGURE 2. First-line antibiotics administered in patients with sickle cell disease who present with fever. $N=27$.

in resource-constrained and malaria-endemic areas. Given the high number of respondents who use oral antibiotics alone to a well-appearing febrile patient, and the ease with which this can be done relative to parenteral antibiotics, the safety and efficacy of this practice should be a priority for evaluation.

Received July 21, 2019. Accepted for publication December 7, 2019.

Published online February 10, 2020.

Acknowledgments: We would like to thank Gladstone Airewele for his critical review of this work.

Financial support: This work was supported by (grants): NIH K12 HL087164 and a Trainee Grant for Patient Safety and Improvement Award, Boston Children's Hospital, and the Baylor Pediatric Pilot Award.

Disclosure: V. N. T. reports personal fees from Norvartis Pharmaceuticals outside the submitted work.

Authors' addresses: Alexandra L. Coria, Maimonides Children's Hospital, E-mail: alexandra.coria@gmail.com. Catherine M. Taylor, Lewisham and Greenwich NHS Trust, London, United Kingdom, E-mail: catamtaylor@gmail.com. Venée N. Tubman, Department of Pediatrics, Baylor College of Medicine, Houston, TX, and Texas Children's Cancer and Hematology Centers, Houston, TX, E-mail: vntubman@txch.org.

\section{REFERENCES}

1. Piel FB, Hay SI, Gupta S, Weatherall DJ, Williams TN, 2013. Global burden of sickle cell anaemia in children under five, 2010-2050: modelling based on demographics, excess mortality, and interventions. PLoS Med 10: e1001484.

2. National Heart, Lung, Blood Institute, 2014. Evidence-Based Management of Sickle Cell Disease. Available at: https://www. nhlbi.nih.gov/health-topics/evidence-based-management-sicklecell-disease. Accessed June 14, 2019.

3. Makani J, Ofori-Acquah SF, Nnodu O, Wonkam A, OheneFrempong K, 2013. Sickle cell disease: new opportunities and challenges in Africa. ScientificWorldJournal 2013: 193252.

4. Ansong D, Akoto AO, Ocloo D, Ohene-Frempong K, 2013. Sickle cell disease: management options and challenges in developing countries. Mediterr J Hematol Infect Dis 5: e2013062.

5. Gill FM, Sleeper LA, Weiner SJ, Brown AK, Bellevue R, Grover R, Pegelow CH, Vichinsky E, 1995. Clinical events in the first decade in a cohort of infants with sickle cell disease. Cooperative study of sickle cell disease. Blood 86: 776-783.

6. Barrett-ConnorE, 1971. Bacterial infection and sickle cell anemia. An analysis of 250 infections in 166 patients and a review of the literature. Medicine (Baltimore) 50: 97-112.

7. Williams TN et al., 2009. Bacteraemia in Kenyan children with sickle-cell anaemia: a retrospective cohort and case-control study. Lancet 374: 1364-1370.

8. Baskin MN, Goh XL, Heeney MM, Harper MB, 2013. Bacteremia risk and outpatient management of febrile patients with sickle cell disease. Pediatrics 131: 1035-1041.

9. World Health Organization, 2002. IMCl Adaptation Guide 2C. Geneva, Switzerland: World Health Organization.

10. Tubman VN, Marshall R, Jallah W, Guo D, Ma C, OheneFrempong K, London WB, Heeney MM, 2016. Newborn screening for sickle cell disease in Liberia: a pilot study. Pediatr Blood Cancer 63: 671-676.

11. Rahimy MC, Gangbo A, Ahouignan G, Alihonou E, 2009. Newborn screening for sickle cell disease in the Republic of Benin. J Clin Pathol 62: 46-48.

12. Tshilolo L, Kafando E, Sawadogo M, Cotton F, Vertongen F, Ferster A, Gulbis B, 2008. Neonatal screening and clinical care programmes for sickle cell disorders in sub-Saharan Africa: lessons from pilot studies. Public Health 122: 933-941.

13. Obaro SK, Iroh Tam PY, 2016. Preventing infections in sickle cell disease: the unfinished business. Pediatr Blood Cancer 63: 781-785.

14. Kizito ME, Mworozi E, Ndugwa C, Serjeant GR, 2007. Bacteraemia in homozygous sickle cell disease in Africa: is pneumococcal prophylaxis justified? Arch Dis Child 92: 21-23.

15. Makani $\mathrm{J}$ et al., 2015. Bacteraemia in sickle cell anaemia is associated with low haemoglobin: a report of 890 admissions to a tertiary hospital in Tanzania. Br J Haematol 171: 273-276.

16. Okuonghae HO, Nwankwo MU, Offor EC, 1993. Pattern of bacteraemia in febrile children with sickle cell anaemia. Ann Trop Paediatr 13: 55-64.

17. Rees DC, Williams TN, Gladwin MT, 2010. Sickle-cell disease. Lancet 376: 2018-2031.

18. McAuley CF et al., 2010. High mortality from Plasmodium falciparum malaria in children living with sickle cell anemia on the coast of Kenya. Blood 116: 1663-1668.

19. Makani $\mathrm{J}$ et al., 2010. Malaria in patients with sickle cell anemia: burden, risk factors, and outcome at the outpatient clinic and during hospitalization. Blood 115: 215-220.

20. Were T, Davenport GC, Hittner JB, Ouma C, Vulule JM, Ong'echa JM, Perkins DJ, 2011. Bacteremia in Kenyan children presenting with malaria. J Clin Microbiol 49: 671-676. 